\title{
HIDRODINÂMICA E DISTRIBUIÇÃO DE SEDIMENTOS EM SUSPENSÃO DOS ESTUÁRIOS DOS RIOS ITAPOCU, TIJUCAS E CAMBORIÚ. [RELATÓRIO]*
}

\author{
SCHETTINI, C.A.F \& J.L.B. CARVALHO \\ CTTMar/UNIVALI
}

\begin{abstract}
RESUMO
Este relatório apresenta os dados obtidos durante campanhas de coleta de dados hidrográficos e sedimentológicos realizadas nos estuários dos rios Itapocu, Tijucas e Camboriú em dezembro 1995. Estas campanhas consistiram de experimentos de 13 horas de duração com aquisição de dados de velocidade e direção de corrente, salinidade e turbidez em intervalos de uma hora em uma estação fixa próxima da desembocadura de cada sistema, e dados de salinidade e turbidez ao longo dos estuários durante os períodos de estofa de baixa e preamar. As informações são fornecidas em tabelas e em gráficos de distribuição do campo dos parâmetros coletados
\end{abstract}

Palavras chave: (1) Rio Itapocu; Rio Tijucas; Rio Camboriú; (2) estuário; (3) hidrodinâmica; (4) material em suspensão.

\section{HYDRODYNAMICS AND SUSPENDED SEDIMENT DISTRIBUTION OF THE ITAPOCU, TIJUCAS AND CAMBORIÚ ESTUARIES. [TECHNICAL REPORT]}

\begin{abstract}
This report presents the data from the hydrographic and sedimentological surveys carried out in the Itapocu, Tijucas and Camboriú estuaries during December, 1995. These surveys were done through 13 hours experiments to record currents, salinity and turbidity hourly in a fixed sampling station near the mouth of each system, and salinity and turbidity data recording along the estuaries during ebb and flood slack waters. The information is presented in tables and field distribution charts of the recorded parameters.
\end{abstract}

Keywords: (1) Itapocu R.; Tijucas R.; Camboriú R.; (2) estuary; (3) hydrodynamic; (4) suspended sediments.

\section{INTRODUÇÃO}

Este trabalho dá continuidade às atividades descritas em Schettini \& Carvalho (neste volume), objetivando fornecer um conhecimento básico das características hidrodinâmicas e sedimentológicas de sistemas estuarinos ao longo do litoral catarinense. Além das campanhas realizadas no estuário do Rio Itajaí-açu, o "Projeto Integrado da Foz do Rio Itajaí-açu e Região Costeira Adjacente: Diagnóstico e Avaliação Ambiental" incluiu também os estuário vizinhos, sendo o do Rio Itapocu ao norte, e os dos rios Camboriú e Tijucas ao sul.

O presente trabalho descreve os resultados de levantamentos de dados hidrográficos e sedimentológicos realizados nos estuários dos rios Itapocu, Tijucas e Camboriú em dezembro de 1995. As informa-

\footnotetext{
*Trabalho realizado com recursos da Fundação Banco do Brasil.
} 
ções obtidas nestes estuários já foram parcialmente divulgadas em artigos e encontros científicos (Schettini et al., 1996a; Schettini et al., 1996b; Jabor et al., 1996a; Jabor et al., 1996b; Schettini \& Klein, 1997a; Schettini \& Klein, 1997b;), sendo que o objetivo deste relatório técnico é disponibilizar os dados obtidos em uma forma reduzida, permitindo assim o seu uso por outros pesquisadores.

\section{CARACTERÍSTICAS GERAIS}

As bacias hidrográficas dos rios Itapocu e Tijucas apresentam a mesma ordem de grandeza, com 2.930 e $2.420 \mathrm{~km}^{2}$, respectivamente (GAPLAN, 1986), e a do Rio

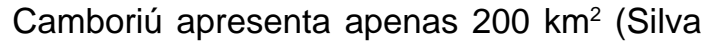
\& Schettini, 1997)(Fig. 1). A descarga fluvial é medida com freqüência diária desde 1940 no Rio Itapocu, na estação linimétrica de Jaraguá do Sul que representa $27 \%$ da área à montante da bacia. No Rio Tijucas, a descarga é monitorada desde 1945 na estação linimétrica de Major que representa $66 \%$ da área à montante da bacia. Extrapolando linearmente a descarga para toda a bacia, estima-se que as descargas médias sejam aproximadamente de 77 e $58 \mathrm{~m}^{3} . \mathrm{s}^{-1}$ para os rio Itapocu e Tijucas, respectivamente. Estas estações linimétricas são atualmente mantidas pela Agencia Nacional de Energia Elétrica (ANEEL). Não existem medições de descarga para o Rio Camboriú, no entanto esta foi estimada entre 3 e $6 \mathrm{~m}^{3} \cdot \mathrm{s}^{-1}$ como média anual (Silva \& Schettini, 1997).

O regime de maré apresenta-se o mesmo para todos os sistemas estuarinos, sendo este descrito em Schettini \& Carvalho (neste volume). Por outro lado, as condições energéticas diferem grandemente para cada desembocadura.

A barra do estuário do Rio Tijucas está localizada em uma planície costeira no interior da Baía de Tijucas, estando abrigada do ataque de ondas pela península de Porto Belo ao norte e pela llha de Santa Catarina e pe- nínsula de Ganchos ao Sul. Isto permite o acúmulo de sedimentos finos exportados pelo estuário gerando assim extensos planos lamosos de maré. O estuário do Rio Itapocu deságua em uma linha de costa desabrigada, de alta energia, possibilitando a formação da Lagoa de Barra Velha atrás de uma ilha barreira bem definida (Figura 2; Schettini \& Klein, 1997a). Diferentemente dos dois anteriores, o estuário do Rio Camboriú deságua na Enseada de Camboriú, margeando um promontório que o torna abrigado e estável (Siegle et al., 1997; Siegle et al., 1998).

Nas bacias dos rios Itapocu e Tijucas desenvolvem-se atividades agropecuárias de modo geral, existindo também importantes pólos industriais, como Jaraguá do Sul na bacia do Itapocu, e o pólo cerâmico em Tijucas. Nesta última é importante ressaltar a atividade de mineração a céu aberto de solos para extração de argila utilizada na indústria cerâmica, o que intensifica a erosão e transporte de sedimentos para o rio e estuário.

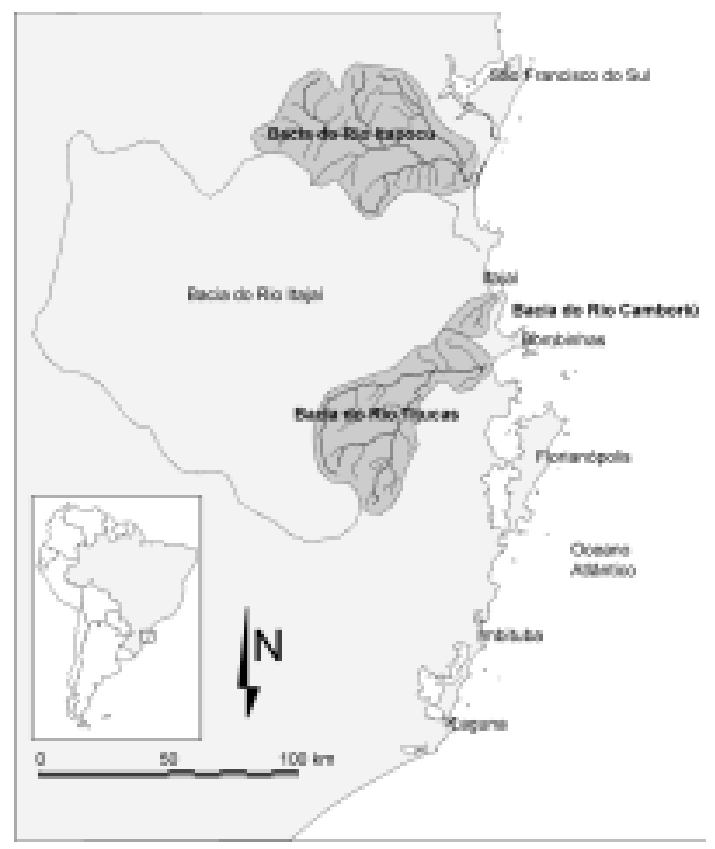

Figura 1: Localização das bacias de drenagens ao longo do litoral de Santa Catarina. 
Embora o estuário do Rio Camboriú seja bastante discreto em termos hidrológicos quando comparado com os outros dois sistemas, ele apresenta uma importância ambiental e econômica extremamente elevada pois deságua na Enseada de Caboriú, e sua drenagem recebe $o$ aporte de canais pluviais e esgotos domésticos da cidade de Balneário Camboriú. A cidade de Balneário Camboriú é o maior centro turístico do sul do Brasil, sendo este diretamente relacionado com a qualidade da água do mar adjacente. Estudos mais recentes já foram realizados para detalhar o conhecimento sobre a hidrodinâmica deste estuário e avaliar o fluxo de sedimentos e poluentes (Franklin-Silva et al., 1998; Siegle et al., 1998A \& B; Schettini et al., 1998).

\section{Material \& Métodos}

Em cada estuário dados de velocidade e direção de corrente, salinidade e turbidez

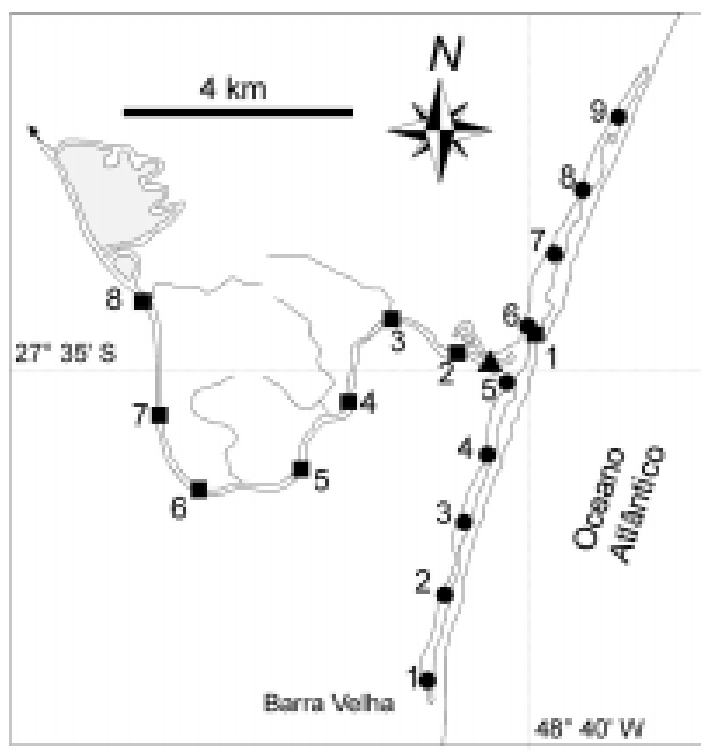

Figura 2: Estuário do Rio Itapocu: s estação de coleta temporal; $n$ estação de coleta no estuário; e l estação de coleta na Lagoa de Barra Velha. foram coletados em uma estação fixa próxima da desembocadura de cada sistema (Figuras 2, 3 e 4) durante experimentos de 12 horas de duração (13 amostras), representando assim aproximadamente um ciclo de maré. Adicionalmente foram realizados perfis longitudinais de salinidade e turbidez durante os períodos de estofa de preamar e baixamar (Figuras 2, 3 e 4), utilizando-se um barco de alumínio com motor de popa.

Todas as campanhas foram realizadas durante condições de maré de quadratura, nos dias 14, 15 e 16 de dezembro de 1995, nos estuários dos Rios Itapocu, Tijucas e Camboriú, respectivamente. As campanhas de coleta de dados foram realizadas em dias consecutivos para se observar o comportamento hidrológico sob as mesmas condições de maré e de regime hidrológico, em termos quasi - sinópticos.

\section{I - Nivel da Água}

Foi utilizada a previsão da maré astronômica para o Porto de Itajaí como informação sobre o nível da água durante os períodos de coleta. Considerando a proximidade

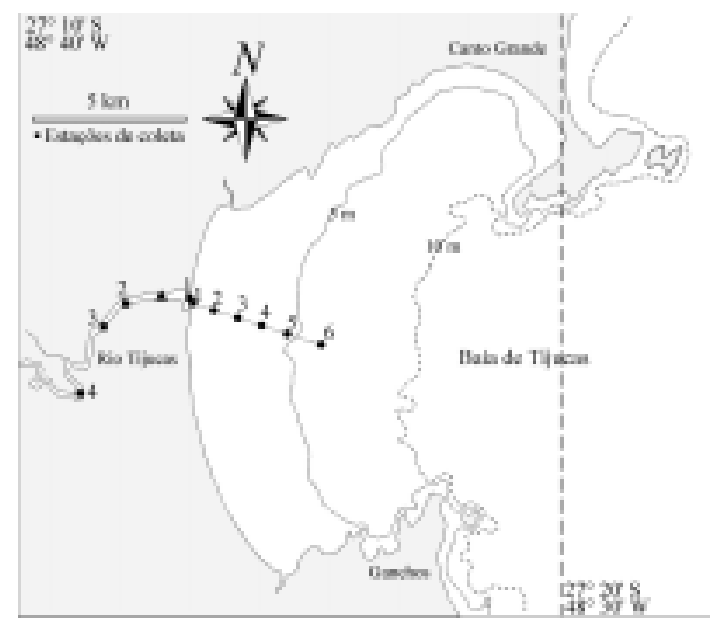

Figura 3: Baía de Tijucas e o estuário do Rio Tijucas: s estação de coleta temporal; n estação de coleta no estuário; I estação de coleta na baía. 
dos estuários deste ponto central e que as condições meteorológicas permaneceram estáveis durante os dias das campanhas, assume-se que a diferença entre a maré prevista e a observada possa ser desprezada.

\section{II - Velocidade e direção de corrente:}

Dados de velocidade e direção de corrente foram obtidos utilizando-se correntógrafos SensorData ${ }^{\mathrm{TM}}$ SD6000, com taxa de aquisição de 5 minutos. No estuário do Rio Itapocu foram fundeados dois correntógrafos no talvegue, com profundidade média de $6 \mathrm{~m}$. Os correntógrafos foram posicionados a $1 \mathrm{~m}$ abaixo da superfície e a $1 \mathrm{~m}$ acima do fundo. Nos estuário do Rio

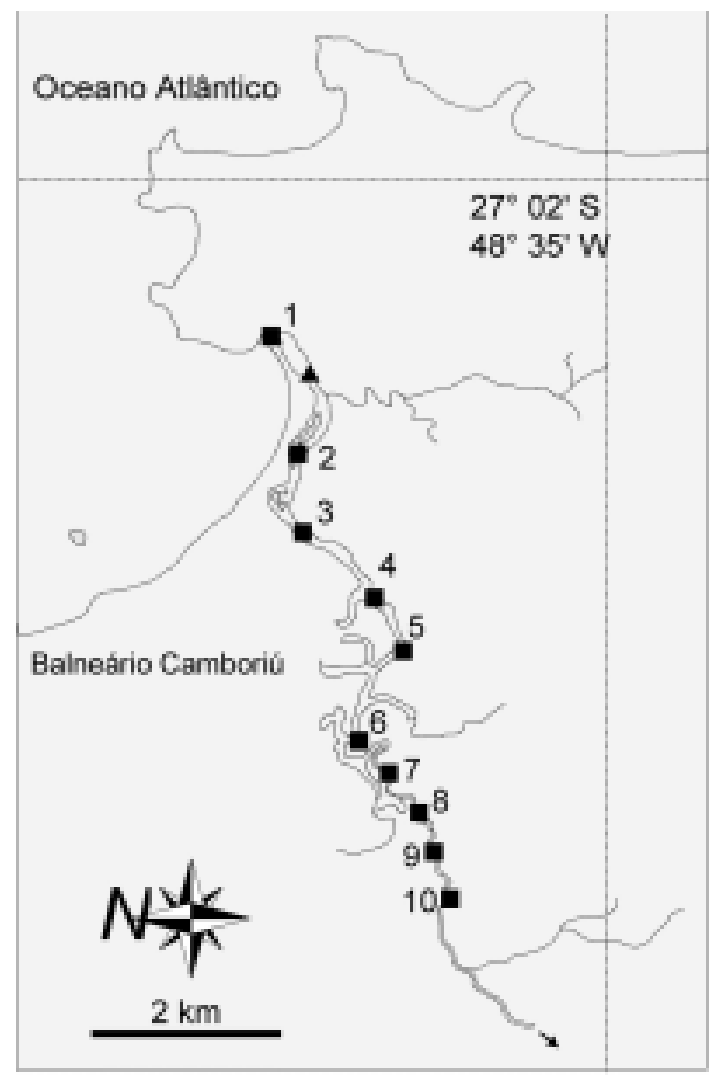

Figura 4: Estuário do Rio Camboriú: s estação de coleta temporal; $n$ estação de coleta no estuário.
Camboriú e do Rio Tijucas, devido à pouca profundidade do local das estações fixas, foi utilizado apenas 1 correntógrafo posicionado à meia água.

Os dados de corrente foram posteriormente integrados para períodos de 25 minutos em torno de cada hora cheia, perfazendo-se assim 13 observações. Posteriormente os dados foram reduzidos para a componente normal à seção transversal, tendo valor negativo para o escoamento com sentido para o mar, e positivo para o escoamento com sentido para o continente.

\section{III - Salinidade e turbidez}

Dados de salinidade e turbidez foram coletados em intervalos de uma hora a cada metro de coluna de água nas estações fixas, e durante os períodos de estofa de preamar e baixa mar ao longo do eixo longitudinal do estuário. A salinidade foi medida com uma sonda multiparâmetro Horiba ${ }^{\mathrm{TM}}$, e a turbidez foi medida com uma sonda OBS3 ${ }^{\circledR}$, pré calibrada para concentrações de 0 até $300 \mathrm{mg.l}$ 1.

No campanha no Rio Itapocu foi realizado um perfil adicional ao longo da Lagoa de Barra Velha (Figura 2), e na campanha no Rio Tijucas foi realizado um perfil adicional da desembocadura do estuário para o centro da Baía de Tijucas (Figura 4).

\section{RESULTADOS}

Os dados coletados na campanha no estuário do Rio Itapocu são apresentados nas Tabelas 1 até 9 , sendo: as Tabelas 1 até 3 são referentes à velocidade de corrente, salinidade e turbidez, respectivamente, da amostragem temporal na estação fixa; as Tabelas 4 e 5 são referentes à salinidade $e$ turbidez respectivamente, no perfil ao longo do eixo longitudinal do canal durante a estofa de preamar; as Tabelas 6 e 7 são referentes à salinidade e turbidez respectivamente, no perfil ao longo do eixo longitudinal do ca- 
Tabela 1: Corrente $\left(\mathrm{cm}^{-1} \mathrm{~s}^{-1}\right)$ ao longo do tempo na estação fixa no estuário do Rio Itapocu.

\begin{tabular}{|c|c|c|c|c|c|c|c|c|c|c|c|c|c|}
\hline $\begin{array}{c}\text { Prof. } \\
\text { (m) }\end{array}$ & 1 & 2 & 3 & 4 & 5 & 6 & 7 & 8 & 9 & 10 & 11 & 12 & 13 \\
\hline-1 & $-1,9$ & $-4,3$ & $-16,5$ & $-19,0$ & $-18,2$ & $-13,9$ & $-11,1$ & $-16,2$ & $-21,4$ & $-24,0$ & $-23,0$ & $-12,7$ & $-2,3$ \\
\hline-4 & 13,7 & 14,1 & 2,1 & $-1,0$ & $-1,0$ & $-1,1$ & $-1,0$ & $-1,0$ & $-1,0$ & $-1,0$ & $-1,1$ & $-1,0$ & 12,7 \\
\hline
\end{tabular}

Tabela 2: Salinidade (\%) ao longo do tempo na estação fixa no estuário do Rio Itapocu.

\begin{tabular}{|c|c|c|c|c|c|c|c|c|c|c|c|c|c|}
\hline $\begin{array}{l}\text { Prof. } \\
(\mathrm{m})\end{array}$ & 1 & 2 & 3 & 4 & 5 & 6 & 7 & 8 & 9 & 10 & 11 & 12 & 13 \\
\hline 0 & 4 & 4 & 5 & 5 & 6 & 5 & 4 & 4 & 4 & 3 & 3 & 3 & 3 \\
\hline-1 & 11 & 14 & 6 & 7 & 8 & 7 & 5 & 16 & 5 & 4 & 10 & 11 & 12 \\
\hline-2 & 23 & 20 & 18 & 21 & 23 & 22 & 17 & 23 & 22 & 19 & 19 & 18 & 23 \\
\hline-3 & 25 & 25 & 23 & 25 & 25 & 24 & 24 & 25 & 24 & 23 & 23 & 21 & 24 \\
\hline-4 & 26 & 26 & 25 & 26 & 26 & 25 & 24 & 26 & 25 & 25 & 25 & 24 & 25 \\
\hline-5 & 26 & 27 & 26 & 26 & 26 & 26 & 25 & 26 & 26 & 26 & 25 & 24 & 25 \\
\hline-6 & 26 & - & - & - & - & - & - & - & - & - & - & - & - \\
\hline
\end{tabular}

Tabela 3: Turbidez (mg.t-1) ao longo do tempo na estação fixa no estuário do Rio Itapocu.

\begin{tabular}{c|ccccccccccccc}
\hline $\begin{array}{c}\text { Prof.( } \\
\mathrm{m})\end{array}$ & 1 & 2 & 3 & 4 & 5 & 6 & 7 & 8 & 9 & 10 & 11 & 12 & 13 \\
\hline 0 & 16 & 22 & 17 & 22 & 17 & 17 & 20 & 27 & 22 & 22 & 24 & 22 & 22 \\
-1 & 19 & 20 & 17 & 24 & 17 & 17 & 19 & 14 & 20 & 22 & 19 & 17 & 16 \\
-2 & 25 & 24 & 19 & 19 & 18 & 16 & 15 & 12 & 12 & 15 & 14 & 22 & 20 \\
-3 & 27 & 26 & 22 & 18 & 18 & 15 & 14 & 14 & 12 & 12 & 11 & 30 & 22 \\
-4 & 29 & 26 & 27 & 20 & 16 & 15 & 12 & 12 & 12 & 11 & 11 & 26 & 27 \\
-5 & 28 & 28 & 27 & 21 & 18 & 15 & 14 & 12 & 21 & 12 & 16 & 30 & 35 \\
-6 & 29 & - & - & - & - & - & - & - & - & - & - & - & - \\
\hline
\end{tabular}

Tabela 4: Salinidade (\%o) no eixo longitudinal do estuário do Rio Itapocu durante a preamar (8:30, 14/12/95).

\begin{tabular}{c|cccccc}
\hline Prof. $(\mathrm{m})$ & $0 \mathrm{~km}$ & $2,3 \mathrm{~km}$ & $3,4 \mathrm{~km}$ & $5,1 \mathrm{~km}$ & $6,8 \mathrm{~km}$ & $8,5 \mathrm{~km}$ \\
\hline 0 & 10 & 3 & 3 & 2 & 2 & 2 \\
-1 & 25 & 10 & 11 & 3 & 2 & 2 \\
-2 & 27 & 21 & 20 & 15 & 21 & 20 \\
-3 & - & 24 & 23 & 23 & 23 & 22 \\
-4 & - & 24 & 24 & 25 & 25 & 24 \\
-5 & - & 26 & 25 & 25 & 25 & 25 \\
-6 & - & - & 25 & & & 25 \\
\hline
\end{tabular}

Tabela 5: Turbidez (mg. $\left.\mathrm{I}^{-1}\right)$ no eixo longitudinal do estuário do Rio Itapocu durante a preamar (8:30, 14/12/95).

\begin{tabular}{c|cccccc}
\hline Prof. $(\mathrm{m})$ & $0 \mathrm{~km}$ & $2,3 \mathrm{~km}$ & $3,4 \mathrm{~km}$ & $5,1 \mathrm{~km}$ & $6,8 \mathrm{~km}$ & $8,5 \mathrm{~km}$ \\
\hline 0 & 21 & 18 & 18 & 17 & 21 & 25 \\
-1 & 28 & 16 & 14 & 18 & 21 & 27 \\
-2 & 31 & 24 & 19 & 10 & 8 & 29 \\
-3 & - & 24 & 21 & 9 & 9 & 8 \\
-4 & - & 26 & 22 & 9 & 10 & 9 \\
-5 & - & 31 & 22 & 10 & 11 & 10 \\
-6 & - & - & 22 & & & 10 \\
\hline
\end{tabular}

Tabela 6: Salinidade (\%) no eixo longitudinal do estuário do Rio Itapocu durante a baixamar (16:00 14/12/95).

\begin{tabular}{c|cccccccc}
\hline Prof. $(\mathrm{m})$ & $0 \mathrm{~km}$ & $2,3 \mathrm{~km}$ & $3,4 \mathrm{~km}$ & $5,1 \mathrm{~km}$ & $6,8 \mathrm{~km}$ & $8,5 \mathrm{~km}$ & $10,3 \mathrm{~km}$ & $12,5 \mathrm{~km}$ \\
\hline 0 & 4 & 3 & 3 & 2 & 1 & 1 & 1 & 0 \\
-1 & 11 & 8 & 3 & 2 & 2 & 1 & 1 & 0 \\
-2 & 12 & 22 & 19 & 13 & 7 & 2 & 1 & 0 \\
-3 & 14 & 25 & 24 & 23 & 23 & 22 & 13 & 21 \\
-4 & 15 & 26 & 25 & 25 & 24 & 24 & 24 & 23 \\
-5 & - & - & 25 & 25 & 25 & 25 & - & 23 \\
-6 & - & - & 26 & - & - & 25 & - & 23 \\
-7 & - & - & 26 & - & - & - & - & - \\
\hline
\end{tabular}


SCHETTINI \& CARVALHO: Hidrodinâmica e sedimento em suspensão em estuários catarinenses.

Tabela 7: Turbidez (mg.l-1) no eixo longitudinal do estuário do Rio Itapocu durante a baixamar (16:00 14/12/95).

\begin{tabular}{c|cccccccc}
\hline Prof. $(\mathrm{m})$ & $0 \mathrm{~km}$ & $2,3 \mathrm{~km}$ & $3,4 \mathrm{~km}$ & $5,1 \mathrm{~km}$ & $6,8 \mathrm{~km}$ & $8,5 \mathrm{~km}$ & $10,3 \mathrm{~km}$ & $12,5 \mathrm{~km}$ \\
\hline 0 & 21 & 25 & 27 & 30 & 26 & 25 & 25 & 28 \\
-1 & 18 & 21 & 25 & 30 & 27 & 28 & 26 & 29 \\
-2 & 18 & 9 & 9 & 14 & 22 & 31 & 28 & 31 \\
-3 & 17 & 11 & 10 & 7 & 7 & 9 & 14 & 8 \\
-4 & 17 & 15 & 12 & 9 & 7 & 8 & 8 & 10 \\
-5 & - & - & 15 & 18 & 10 & 8 & - & 12 \\
-6 & - & - & 16 & - & - & 10 & - & 14 \\
-7 & - & - & 21 & - & - & - & - & - \\
\hline
\end{tabular}

Tabela 8: Salinidade (\%) ao longo da Lagoa de Barra Velha. Distância negativa indica as estações no braço norte, e as positivas indica as estações no braço sul.

\begin{tabular}{c|ccccccccc}
\hline Prof. $(\mathrm{m})$ & $-4 \mathrm{~km}$ & $-2,8 \mathrm{~km}$ & $-1,7 \mathrm{~km}$ & $0 \mathrm{~km}$ & $1,1 \mathrm{~km}$ & $2,3 \mathrm{~km}$ & $3,4 \mathrm{~km}$ & $4,6 \mathrm{~km}$ & $5,7 \mathrm{~km}$ \\
\hline 0 & 21 & 20 & 18 & 20 & 7 & 10 & 16 & 20 & 21 \\
-1 & 21 & 20 & 19 & 20 & 12 & 21 & 21 & 21 & 21 \\
-2 & - & - & - & - & 24 & - & - & - & 21 \\
\hline
\end{tabular}

Tabela 9: Turbidez (mg. $\mathrm{l}^{-1}$ ) ao longo da Lagoa de Barra Velha. Distância negativa indica as estações no braço norte, e as positivas indica as estações no braço sul.

\begin{tabular}{c|ccccccccc}
\hline Prof. $(\mathrm{m})$ & $-4 \mathrm{~km}$ & $-2,8 \mathrm{~km}$ & $-1,7 \mathrm{~km}$ & $0 \mathrm{~km}$ & $1,1 \mathrm{~km}$ & $2,3 \mathrm{~km}$ & $3,4 \mathrm{~km}$ & $4,6 \mathrm{~km}$ & $5,7 \mathrm{~km}$ \\
\hline 0 & 4 & 9 & 9 & 14 & 17 & 15 & 15 & 15 & 16 \\
-1 & 5 & 9 & 18 & 14 & 17 & 27 & 16 & 16 & 17 \\
-2 & - & - & - & - & 22 & - & - & - & 17 \\
\hline
\end{tabular}

Tabela 10: Corrente $\left(\mathrm{cm}^{-1} \mathrm{~s}^{-1}\right)$ ao longo do tempo na estação fixa no estuário do Rio Tijucas.

\begin{tabular}{c|ccccccccccccc}
\hline $\begin{array}{c}\text { Prof. } \\
(\mathrm{m})\end{array}$ & 1 & 2 & 3 & 4 & 5 & 6 & 7 & 8 & 9 & 10 & 11 & 12 & 13 \\
\hline-1 & 14 & 8 & 4 & 22 & 12 & 10 & 26 & 11 & 15 & 16 & 3 & 0 & -1 \\
\hline
\end{tabular}

nal durante a estofa de baixamar. As tabelas 8 e 9 apresentam os dados de salinidade e turbidez respectivamente, coletados ao longo da Lagoa de Barra Velha. Todos os valores de distâncias consideram o zero como sendo na desembocadura do sistema.

Os dados coletados na campanha no estuário do Rio Tijucas, são apresentados nas Tabelas 10 a 18, sendo: as Tabelas 10 até 12 são referentes à velocidade de corrente, salinidade e turbidez, respectivamente, da amostragem temporal na estação fixa; as Tabelas 13 e 14 são referentes à salinidade $e$ turbidez respectivamente, no perfil ao longo do eixo longitudinal do canal durante a estofa de preamar; as Tabelas 15 e 16 são referentes à salinidade e turbidez respectivamente, no perfil ao longo do eixo longitudinal do canal durante a estofa de baixamar. As tabe- las 17 e 18 apresentam os dados coletados no perfil para o interior da Baía de Tijucas.

Os dados coletados na campanha no estuário do Rio Camboriú são apresentados nas Tabelas 19 a 25, sendo: as Tabelas 19 até 21 são referentes à velocidade de corrente, salinidade e turbidez, respectivamente, da amostragem temporal na estação fixa; as Tabelas 22 e 23 são referentes à salinidade e turbidez, respectivamente, no perfil ao longo do eixo longitudinal do canal durante a estofa de preamar; e finalmente, as Tabelas 24 e 25 são referentes à salinidade e turbidez, respectivamente, no perfil longitudinal do canal durante a estofa de baixa mar.

Os campos de distribuição vertical/temporal e vertical/longitudinal dos parâmetros coletados são apresentados nas Figuras 5 até 15. 
Tabela 11: Salinidade (\%) ao longo do tempo na estação fixa no estuário do Rio Tijucas.

\begin{tabular}{c|ccccccccccccc}
\hline $\begin{array}{c}\text { Prof. } \\
(\mathrm{m})\end{array}$ & 1 & 2 & 3 & 4 & 5 & 6 & 7 & 8 & 9 & 10 & 11 & 12 & 13 \\
\hline 0 & 1 & 1 & 1 & 1 & 1 & 1 & 1 & 1 & 1 & 1 & 1 & 1 & 1 \\
-1 & 19 & 12 & 4 & 2 & 3 & 2 & 5 & 4 & 7 & 3 & 3 & 2 & 1 \\
$-1,5$ & 26 & 26 & - & - & 17 & 18 & 14 & 15 & 15 & 10 & 14 & 9 & 7 \\
\hline
\end{tabular}

Tabela 12: Turbidez (mg.t-1) ao longo do tempo na estação fixa no estuário do Rio Tijucas.

\begin{tabular}{c|ccccccccccccc}
\hline $\begin{array}{c}\text { Prof. } \\
(\mathrm{m})\end{array}$ & 1 & 2 & 3 & 4 & 5 & 6 & 7 & 8 & 9 & 10 & 11 & 12 & 13 \\
\hline 0 & 85 & 75 & 68 & 65 & 65 & 67 & 83 & 68 & 69 & 74 & 69 & 75 & 70 \\
-1 & 74 & 55 & 59 & 61 & 32 & 58 & 34 & 46 & 59 & 59 & 39 & 39 & 65 \\
$-1,5$ & - & - & - & - & 38 & - & 78 & 12 & 9 & - & 49 & - & 89 \\
\hline
\end{tabular}

Tabela 13: Salinidade (\%o) ao longo do eixo longitudinal do estuário do Rio Tijucas durante a preamar (8:30 15/12/95).

\begin{tabular}{c|cccc}
\hline Prof. $(\mathrm{m})$ & $0 \mathrm{~km}$ & $4 \mathrm{~km}$ & $7 \mathrm{~km}$ & $14 \mathrm{~km}$ \\
\hline 0 & 3 & 1 & 0 & 0 \\
-1 & 3 & 1 & 0 & 0 \\
-2 & - & 10 & 3 & 1 \\
-3 & - & 25 & 16 & 5 \\
-4 & - & - & 18 & - \\
\hline
\end{tabular}

Tabela 14: Turbidez (mg.l-1) ao longo do eixo longitudinal do estuário do Rio Tijucas durante a preamar (8:30 15/12/95).

\begin{tabular}{c|cccc}
\hline Prof. $(\mathrm{m})$ & $0 \mathrm{~km}$ & $4 \mathrm{~km}$ & $7 \mathrm{~km}$ & $14 \mathrm{~km}$ \\
\hline 0 & 59 & 70 & 84 & 92 \\
-1 & 55 & 76 & 77 & 107 \\
-2 & - & 65 & 47 & 86 \\
-3 & - & 201 & 49 & 89 \\
-4 & - & - & 65 & - \\
\hline
\end{tabular}

Tabela 15: Salinidade (\%o) ao longo do eixo longitudinal do estuário do Rio Tijucas durante a baixamar (17:00 15/12/95).

\begin{tabular}{c|cccc}
\hline Prof. $(\mathrm{m})$ & $0 \mathrm{~km}$ & $4 \mathrm{~km}$ & $7 \mathrm{~km}$ & $14 \mathrm{~km}$ \\
\hline 0 & 3 & 1 & 0 & 0 \\
-1 & 10 & 1 & 0 & 0 \\
-2 & - & 14 & 1 & 0 \\
-3 & - & - & 22 & - \\
\hline
\end{tabular}

Tabela 16: Turbidez (mg.t-1) ao longo do eixo longitudinal do estuário do Rio Tijucas durante a preamar (17:00 15/12/95).

\begin{tabular}{c|cccc}
\hline Prof. $(\mathrm{m})$ & $0 \mathrm{~km}$ & $4 \mathrm{~km}$ & $7 \mathrm{~km}$ & $14 \mathrm{~km}$ \\
\hline 0 & 104 & 93 & 81 & 105 \\
-1 & 148 & 88 & 87 & 94 \\
-2 & - & 24 & 92 & 87 \\
-3 & - & - & 28 & - \\
\hline
\end{tabular}


SCHETTINI \& CARVALHO: Hidrodinâmica e sedimento em suspensão em estuários catarinenses.

Tabela 17: Salinidade (\%o) ao longo do perfil na Baía de Tijucas.

\begin{tabular}{c|ccccccc}
\hline Prof. $(\mathrm{m})$ & $0 \mathrm{~km}$ & $0,5 \mathrm{~km}$ & $0,89 \mathrm{~km}$ & $0,9 \mathrm{~km}$ & $1,9 \mathrm{~km}$ & $2,8 \mathrm{~km}$ & $3,8 \mathrm{~km}$ \\
\hline 0 & 3 & 27 & 24 & 29 & 28 & 29 & 29 \\
-1 & 3 & 28 & 27 & 28 & 28 & 28 & 28 \\
-2 & - & 28 & 27 & 28 & 28 & 28 & 28 \\
-3 & - & - & 28 & 28 & 28 & 28 & 28 \\
-4 & - & - & - & - & 28 & 28 & 28 \\
-5 & - & - & - & - & - & 28 & 28 \\
$-5,5$ & - & - & - & - & - & - & 29 \\
\hline
\end{tabular}

Tabela 18: Turbidez (mg.l-1) ao longo do perfil na Baía de Tijucas.

\begin{tabular}{c|ccccccc}
\hline Prof. $(\mathrm{m})$ & $0 \mathrm{~km}$ & $0,5 \mathrm{~km}$ & $0,89 \mathrm{~km}$ & $0,9 \mathrm{~km}$ & $1,9 \mathrm{~km}$ & $2,8 \mathrm{~km}$ & $3,8 \mathrm{~km}$ \\
\hline 0 & 59 & 41 & 31 & 25 & 14 & 10 & 6 \\
-1 & 55 & 42 & 26 & 25 & 14 & 9 & 7 \\
-2 & - & 156 & 29 & 30 & 10 & 6 & 6 \\
-3 & - & - & 59 & 69 & 89 & 18 & 6 \\
-4 & - & - & - & - & 120 & 65 & 12 \\
-5 & - & - & - & - & - & 89 & 37 \\
$-5,5$ & - & - & - & - & - & - & 50 \\
\hline
\end{tabular}

Tabela 19: Corrente $\left(\mathrm{cm}^{\mathrm{s}} \mathrm{s}^{-1}\right)$ ao longo do tempo na estação fixa no estuário do Rio Camboriú.

\begin{tabular}{|c|c|c|c|c|c|c|c|c|c|c|c|c|c|}
\hline $\begin{array}{c}\text { Prof. } \\
(\mathrm{m})\end{array}$ & 1 & 2 & 3 & 4 & 5 & 6 & 7 & 8 & 9 & 10 & 11 & 12 & 13 \\
\hline-1 & 19 & 18 & 22 & 10 & -1 & -10 & -12 & -10 & -16 & -9 & -13 & -7 & 20 \\
\hline
\end{tabular}

Tabela 20: Salinidade (\%o) ao longo do tempo na estação fixa no estuário do Rio Camboriú.

\begin{tabular}{|c|c|c|c|c|c|c|c|c|c|c|c|c|c|}
\hline $\begin{array}{l}\text { Prof. } \\
(\mathrm{m})\end{array}$ & 1 & 2 & 3 & 4 & 5 & 6 & 7 & 8 & 9 & 10 & 11 & 12 & 13 \\
\hline 0 & 16 & 18 & 24 & 25 & 20 & 21 & 21 & 19 & 18 & 18 & 18 & 18 & 20 \\
\hline-1 & 18 & 25 & 25 & 26 & 25 & 23 & 23 & 21 & 20 & 21 & 19 & 18 & 20 \\
\hline$-1,5$ & 25 & 26 & 26 & 26 & 26 & 26 & 26 & 25 & 25 & 25 & 24 & 22 & 22 \\
\hline
\end{tabular}

Tabela 21: Turbidez (mg.l-1) ao longo do tempo na estação fixa no estuário do Rio Camboriú.

\begin{tabular}{c|ccccccccccccc}
\hline $\begin{array}{c}\text { Prof. } \\
(\mathrm{m})\end{array}$ & 1 & 2 & 3 & 4 & 5 & 6 & 7 & 8 & 9 & 10 & 11 & 12 & 13 \\
\hline 0 & 32 & 30 & 22 & 21 & 31 & 27 & 35 & 47 & 49 & 48 & 55 & 55 & 46 \\
-1 & 30 & 18 & 18 & 16 & 15 & 14 & 47 & 44 & 51 & 48 & 50 & 63 & 48 \\
$-1,5$ & 24 & 22 & 18 & 14 & 15 & 25 & 15 & 24 & 39 & 22 & 28 & 34 & 37 \\
\hline
\end{tabular}

Tabela 22: Salinidade (\%) ao longo do eixo longitudinal do estuário do Rio Camboriú durante a preamar (11:00 16/12/ 95).

\begin{tabular}{|c|c|c|c|c|c|c|c|c|c|c|}
\hline $\begin{array}{c}\text { Prof. } \\
\text { (m) }\end{array}$ & $0 \mathrm{~km}$ & $1,5 \mathrm{~km}$ & $2,6 \mathrm{~km}$ & $3,5 \mathrm{~km}$ & $4,5 \mathrm{~km}$ & $5,4 \mathrm{~km}$ & $6 \mathrm{~km}$ & $7,3 \mathrm{~km}$ & $7,8 \mathrm{~km}$ & $8,2 \mathrm{~km}$ \\
\hline 0 & 26 & 17 & 17 & 14 & 8 & 8 & 5 & 4 & 2 & 1 \\
\hline-1 & 26 & 17 & 18 & 14 & 14 & 10 & 10 & 9 & 3 & 1 \\
\hline-2 & 26 & 25 & & 18 & 18 & 16 & 15 & 12 & 10 & 3 \\
\hline-3 & 27 & - & - & 19 & - & 17 & - & - & - & - \\
\hline-4 & 27 & - & - & 20 & - & 18 & - & - & - & - \\
\hline
\end{tabular}


Tabela 23: Turbidez (mg. $\mathrm{I}^{-1}$ ) ao longo do eixo longitudinal do estuário do Rio Camboriú durante a preamar (11:00 16/12/ 95).

\begin{tabular}{c|cccccccccc}
\hline $\begin{array}{c}\text { Prof. } \\
(\mathrm{m})\end{array}$ & $0 \mathrm{~km}$ & $1,5 \mathrm{~km}$ & $2,6 \mathrm{~km}$ & $3,5 \mathrm{~km}$ & $4,5 \mathrm{~km}$ & $5,4 \mathrm{~km}$ & $6 \mathrm{~km}$ & $7,3 \mathrm{~km}$ & $7,8 \mathrm{~km}$ & $8,2 \mathrm{~km}$ \\
\hline 0 & 10 & 53 & 44 & 37 & 28 & 27 & 26 & 32 & 31 & 38 \\
-1 & 11 & 27 & 31 & 46 & 34 & 25 & 30 & 55 & 42 & 35 \\
-2 & 11 & 24 & & 48 & 35 & 27 & 30 & 48 & 46 & 28 \\
-3 & 14 & - & - & 53 & - & 28 & - & - & - & - \\
-4 & 20 & - & - & 60 & - & 29 & - & - & - & - \\
\hline
\end{tabular}

Tabela 24: Salinidade (\%) ao longo do eixo longitudinal do estuário do Rio Camboriú durante a baixamar (17:00 16/12/ 95).

\begin{tabular}{c|cccccccc}
\hline Prof. $(\mathrm{m})$ & $0 \mathrm{~km}$ & $1,5 \mathrm{~km}$ & $2,6 \mathrm{~km}$ & $3,5 \mathrm{~km}$ & $4,5 \mathrm{~km}$ & $5,4 \mathrm{~km}$ & $6 \mathrm{~km}$ & $8,2 \mathrm{~km}$ \\
\hline 0 & 21 & 16 & 19 & 11 & 9 & 7 & 5 & 4 \\
-1 & 21 & 18 & 19 & 13 & 10 & 11 & 10 & 4 \\
-2 & 24 & 22 & & 16 & - & 16 & 14 & 12 \\
-3 & 26 & - & - & 18 & - & 17 & - & - \\
-4 & - & - & - & - & - & 18 & - & - \\
\hline
\end{tabular}

Tabela 25: Turbidez (mg.l-1) ao longo do eixo longitudinal do estuário do Rio Camboriú durante a baixamar (17:00 16/12/ 95).

\begin{tabular}{c|cccccccc}
\hline Prof. $(\mathrm{m})$ & $0 \mathrm{~km}$ & $1,5 \mathrm{~km}$ & $2,6 \mathrm{~km}$ & $3,5 \mathrm{~km}$ & $4,5 \mathrm{~km}$ & $5,4 \mathrm{~km}$ & $6 \mathrm{~km}$ & $8,2 \mathrm{~km}$ \\
\hline 0 & 49 & 68 & 40 & 42 & 38 & 36 & 27 & 37 \\
-1 & 47 & 56 & 44 & 44 & 48 & 38 & 38 & 58 \\
-2 & 40 & 57 & & 42 & - & 27 & 40 & 53 \\
-3 & 48 & - & - & 45 & - & 27 & - & - \\
-4 & - & - & - & - & - & 28 & - & -
\end{tabular}

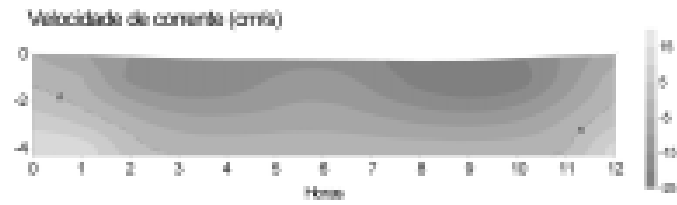

Solindode $(0 / 00)$

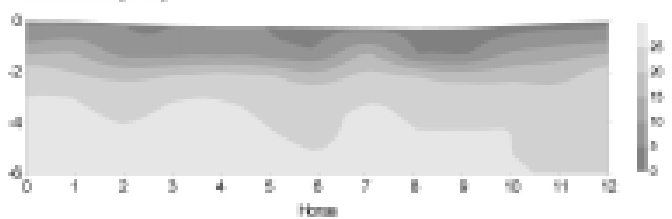

Turbine imgl|

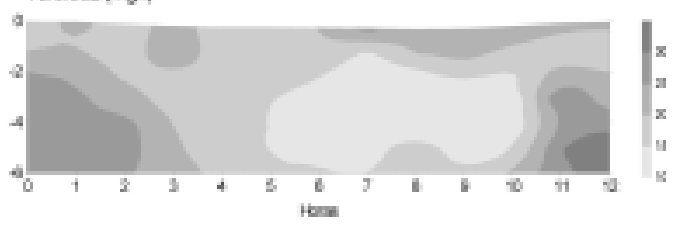

Figura 5: Distribuição temporal/vertical do campo de corrente $\left(\mathrm{cm} . \mathrm{s}^{-1}\right)$, salinidade $(\%)$, e turbidez $\left(\mathrm{mg}^{-1} \mathrm{I}^{-1}\right)$, no estuário do Rio Itapocu.

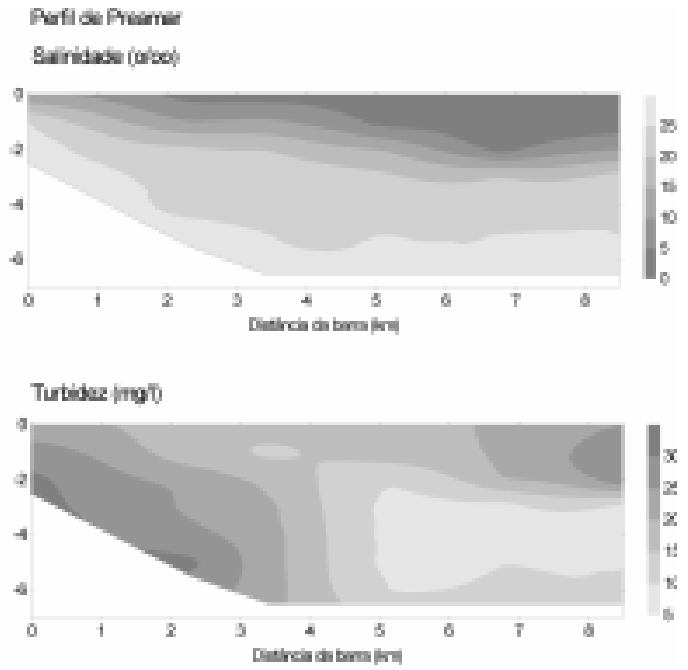

Figura 6: Distribuição longitudinal do campo de salinidade (\%) e turbidez (mg. $\mathrm{I}^{-1}$ ), no estuário do Rio Itapocu durante a preamar. 


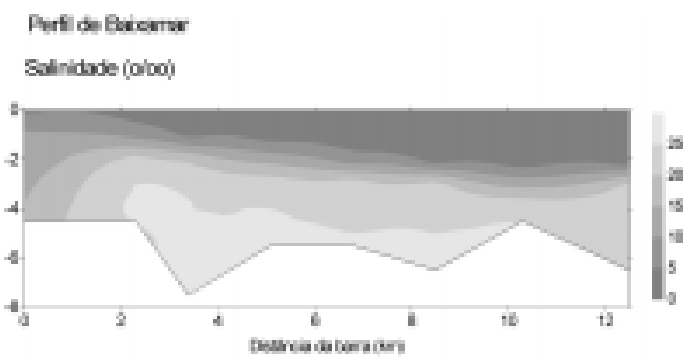

Turtidez (mol)

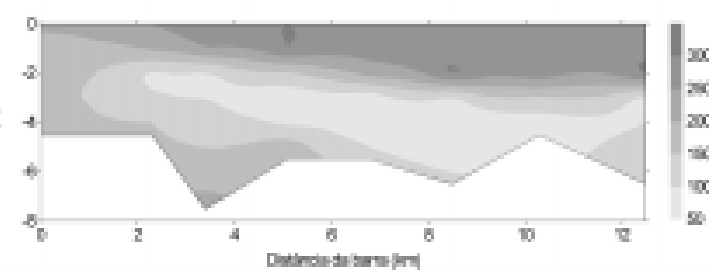

Figura 7: Distribuição longitudinal do campo de salinidade $(\%)$ e turbidez (mg..$\left.^{-1}\right)$, no estuário do Rio Itapocu durante a baixamar.
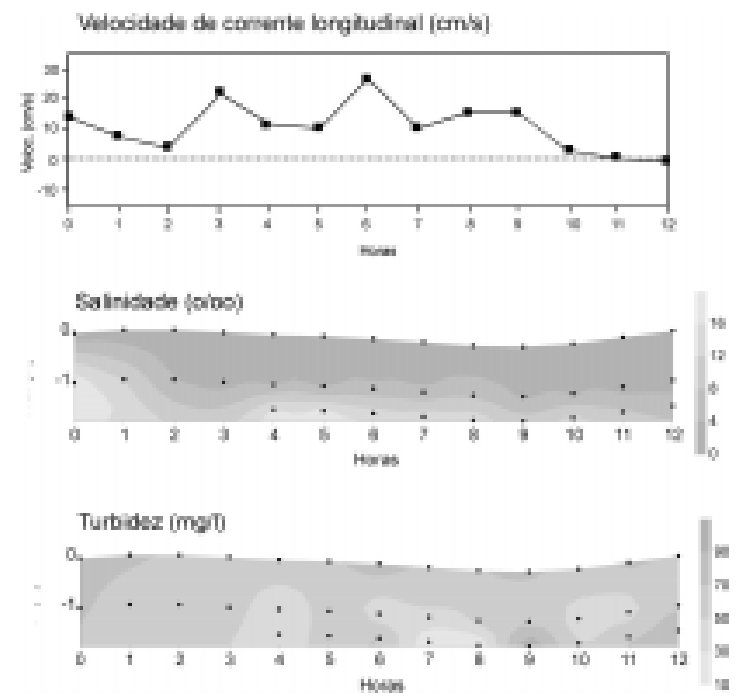

Figura 9: Variação temporal do campo de corrente $\left(\mathrm{cm} . \mathrm{s}^{-1}\right)$, e distribuição temporal/espacial do campo de salinidade (\%) e turbidez (mg.l-1 $)$, no estuário do Rio Tijucas.
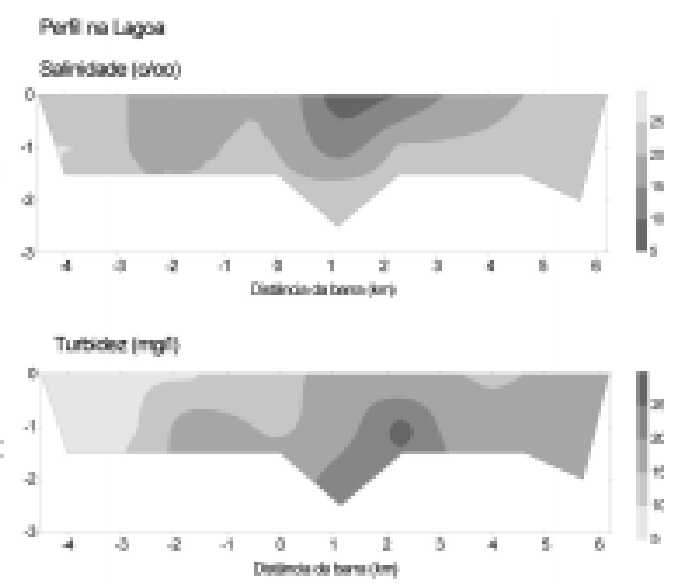

Figura 8: Distribuição longitudinal do campo de salinidade (\%) e turbidez $\left(\mathrm{mg}^{-\mathrm{I}^{-1}}\right)$, no lagoa de Barra Velha.

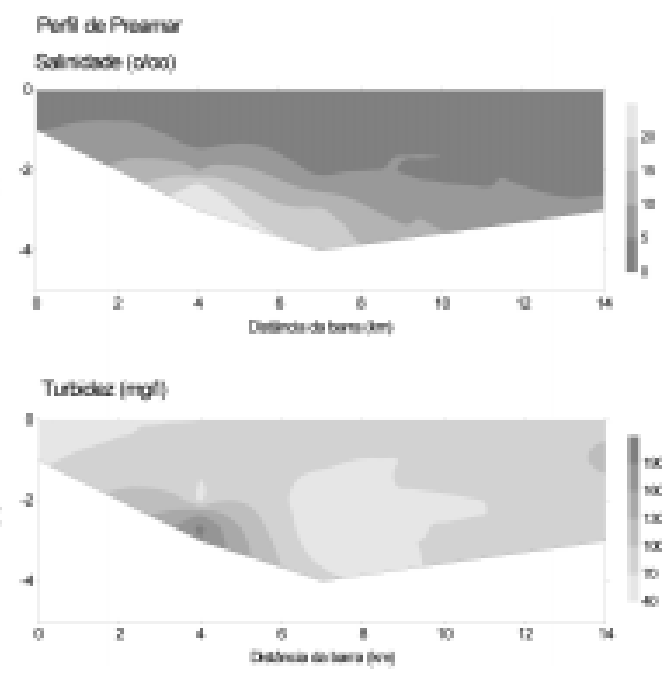

Figura 10: Distribuição longitudinal do campo de salinidade (\%) e turbidez (mg..$\left.^{-1}\right)$, no estuário do Rio Tijucas durante a preamar. 


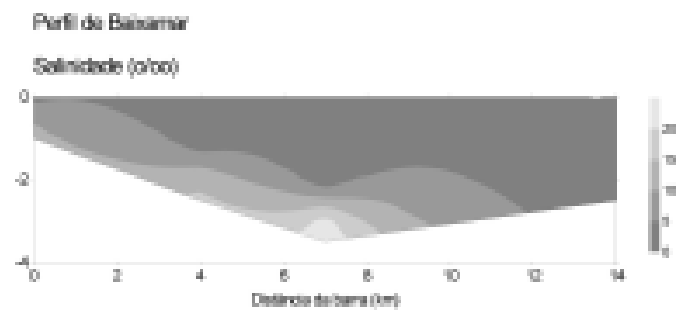

Turtides $/ m g^{2}$

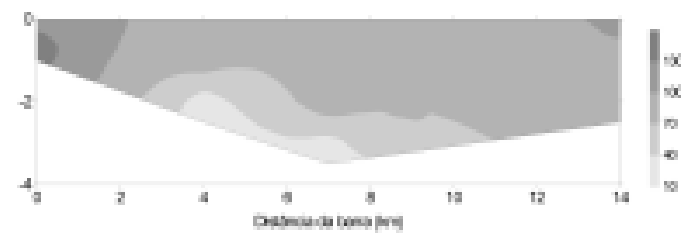

Figura 11: Distribuição longitudinal do campo de salinidade (\%) e turbidez (mg. $\mathrm{I}^{-1}$ ), no estuário do Rio Tijucas durante a baixamar.

Weloodase de corrembe (lem/s)

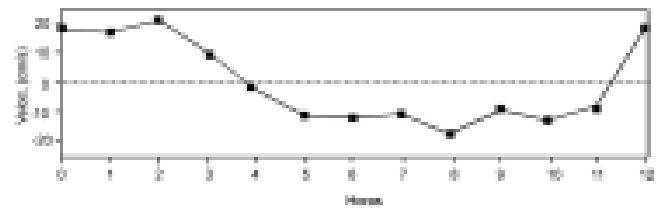

Salrifade (abo)
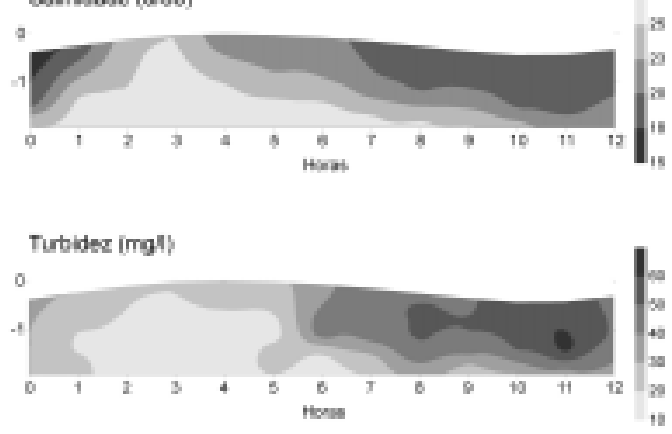

Figura 13: Variação temporal do campo de corrente $(\mathrm{cm} . \mathrm{s}$ 1), e distribuição temporal/espacial do campo de salinidade (\%) e turbidez (mg. $\left.\mathrm{I}^{-1}\right)$, no estuário do Rio Camboriú.
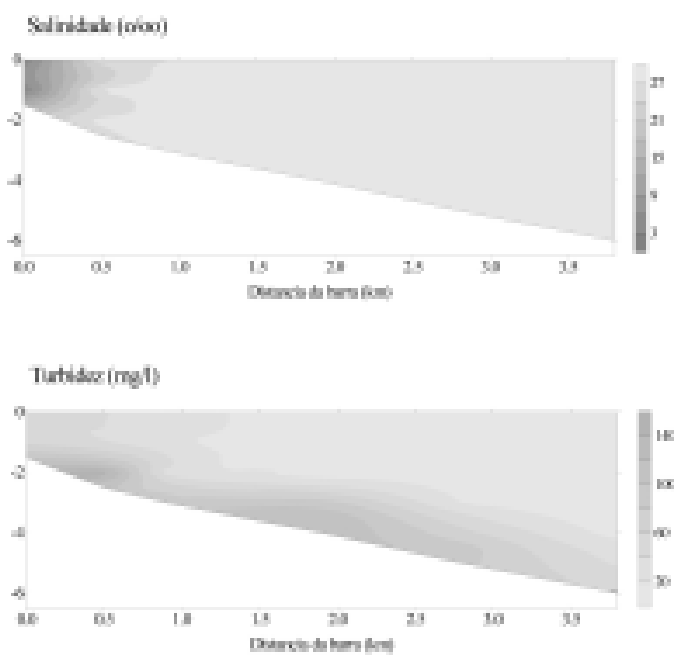

Figura 12: Distribuição longitudinal do campo de salinidade (\%o) e turbidez $\left(\mathrm{mg}^{\mathrm{I}} \mathrm{I}^{-1}\right)$, na Baía de Tijucas.

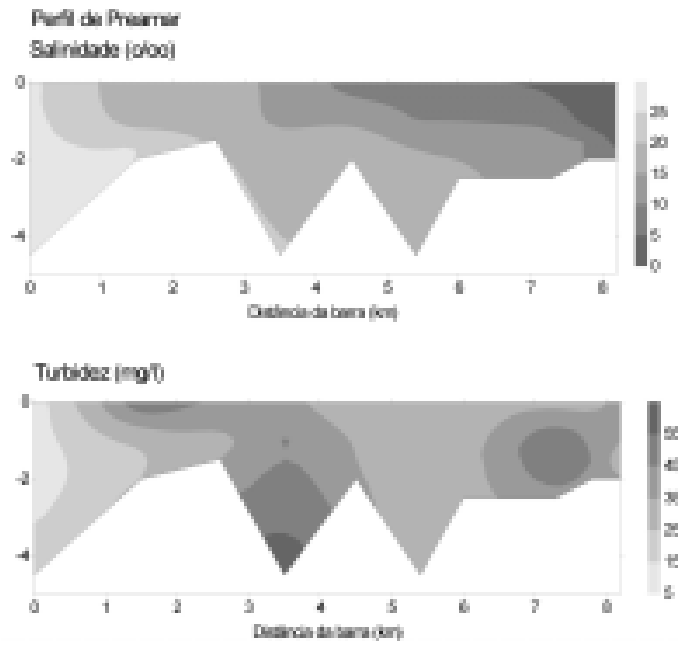

Figura 14: Distribuição longitudinal do campo de salinidade (\%) e turbidez (mg.l-1), no estuário do Rio Camboriú durante a preamar. 


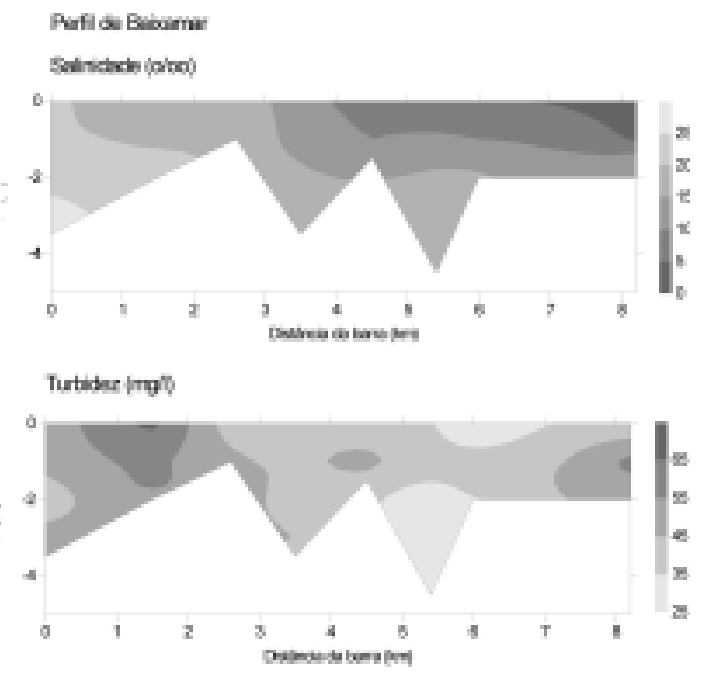

Figura 15: Distribuição longitudinal do campo de salinidade (\%) e turbidez (mg.l-1 $)$, no estuário do Rio Camboriú durante a baixamar.

\section{AGRADECIMENTOS}

Os autores agradecem à Pablo Jabor pelo auxílio prestado nas atividade de coleta de dados; à Eduardo Siegle pela revisão crítica do manuscrito; e à Fundação Banco do Brasil pelos recursos disponibilizados para a execução deste projeto.

\section{REFERÊNCIAS BIBLIOGRÁFICAS}

Jabor, P.M.; Carvalho, J.L.B.; Schettini, C.A.F. 1996a Estuários do litoral centro norte de Santa Catarina: aspectos hidrodinâmicos. SEMANA NACIONAL DE OCEANOGRAFIA, 9, Arraial do Cabo, Resumos... Uerj, p27.

Jabor, P.M.; Carvalho, J.L.B.; Schettini, C.A.F. 1996b Estuários do litoral centro norte de Santa Catarina: aspectos hidrodinâmicos. SEMINÁRIO INTEGRADO DE INICIA-
ÇÃO CIENTÍFICA, 2, Chapecó, Resumos... Univali/Furb/Unoesc, p38.

Franklin-Silva, L.; Schettini, C.A.F.; Siegle, E. 1998 Variabilidade lateral da salinidade no estuário do Rio Camboriú. In: SEMANA NACIONAL DE OCEANOGRAFIA, 11 , Rio Grande, Resumos Expandidos...Furg, pp419-421.

GAPLAN 1987 Atlas de Santa Catarina. 173pp.

Schettini, C.A.F.; Carvalho, J.L.B.; Jabor, P. 1996a Comparative hydrology and suspended matter distribution of four estuaries in Santa Catarina State Southern Brazil. WORKSHOP ON COMPARATIVE STUDIES OF TEMPERATE COAST ESTUARIES, Bahía Blanca, Argentina, Proceedings... UNS/IADO/NSF/IAI, pp29-32.

Schettini, C.A.F.; Carvalho, J.L.B.; Jabor, P. 1996b Hidrologia e distribuição de material em suspensão de quatro estuários de Santa Catarina. SIMPÓSIO SOBRE OCEANOGRAFIA, 3, São Paulo, Resumos... IO-USP, p333.

Schettini, C.A.F. \& Klein, A.H.F. 1997a Processos costeiros e dinâmica estuarina: um modelo para o estuário do Rio Itapocu, Santa Catarina. CONGRESSO DA ASSOCIAÇÃO BRASILEIRA DE ESTUDOS DO QUATERNÁRIO, 6, Curitiba, Resumos Expandidos... ABEQUA, pp127-131.

Schettini, C.A.F. \& Klein, A.H.F. 1997b Aspectos da dinâmica sedimentar da Baía de Tijucas. SEMANA NACIONAL DE OCEANOGRAFIA, 10, Itajaí, Resumos Expandidos...FACIMAR/UNIVALI, pp493495.

Schettini, C.A.F.; Barreiros, M.; Siegle, E. 1998 Transporte e distribuição de coliformes fecais no estuário do Rio Camboriú - SC. In: SEMANA NACIONAL DE OCEANOGRAFIA, 11, Rio Grande, Resumos Expandidos...Furg, pp410-412.

Siegle, E.; Klein, A.H.F.; Toldo Jr., E.E.; Schettini, C.A.F. 1997 Análise de dados 
pretéritos da desembocadura do Rio Camboriú, Balneário Camboriú - SC. SIMPÓSIO BRASILEIRO DE RECURSOS HÍDRICOS, 12, Vitória, Anais...ABRH, pp603-610.

Siegle, E.; Schettini, C.A.F; Klein, A.H.F.; Toldo Jr., E.E. 1998a Mecanismos de transporte de sedimentos em suspensão no estuário do Rio Camboriú, Balneário Camboriú, Santa Catarina. In: ENCONTRO DE ENGENHARIA DE SEDIMENTOS, 3, Belo Horizonte, Anais... ABRH, p25-32.

Siegle, E.; Schettini, C.A.F.; Klein, A.; Toldo Jr., E.E. 1998b Estuário do Rio Camboriú, Balneário Camboriú, SC: hidrografia e se- dimentos em suspensão. In: SEMANA NACIONAL DE OCEANOGRAFIA, 11, Rio Grande, Resumos Expandidos...Furg, pp463-465.

Siegle, E.; Klein, A.; Toldo Jr., E.E.; Abreu, J.G.N. 1998 Morphology and stability of the Camboriu River inlet, Santa Catarina, Brazil. Journal of Coastal Research, 26(SI):94-100.

Silva, L.F. \& Schettini, C.A.F. 1997 Contribuição à hidrologia do estuário do Rio Camboriú: avaliação da contribuição fluvial. SEMANA NACIONAL DE OCEANOGRAFIA, 10, Itajaí, Resumos Expandidos...FACIMAR/UNIVALI, pp496498. 